\title{
Pioglitazone Ameliorates Endothelial Dysfunction in Those with Impaired Glucose Regulation among the First-Degree Relatives of Type 2 Diabetes Mellitus Patients
}

\author{
Xuemei Yu Peihong Chen Hongtao Wang Tong Zhu \\ Department of Endocrinology, Fengxian Central Hospital, Shanghai, P.R. China
}

\section{Key Words}

Impaired glucose regulation - Endothelial dysfunction •

Pioglitazone

\begin{abstract}
Objective: To study the effects of pioglitazone on endothelial dysfunction of subjects with impaired glucose regulation (IGR) among the first-degree relatives of patients with type 2 diabetes mellitus (T2DM). Subjects and Methods: The firstdegree relatives of T2DM patients were screened with oral glucose test and IGR was diagnosed. IGR subjects whose blood glucose was still above the level after 1-month exercise were randomized to receive pioglitazone ( $15 \mathrm{mg} /$ day) or vehicle for 12 weeks. Endothelial function was assessed as endothelium-dependent and -independent vasodilation. Blood nitric oxide (NO), blood pressure, body mass index, insulin and serum lipids were also measured. Area under the curve of glucose ( $A \cup C_{\text {glu }}$ ) and insulin ( $A \cup C_{I N S}$ ), homeostasis model assessment of insulin resistance (HOMA-IR), HOMA of $\beta$-cell function (HOMA- $\beta$ ) and early insulin secretion index $\left(\Delta \mathrm{I}_{30} / \Delta \mathrm{G}_{30}\right)$ were calculated. Results: After pioglitazone treatment, fasting plasma, 2-hour plasma glucose, triglyceride (TG), fasting insulin, $A \cup C_{\text {glu }}$, HOMA- $\beta$ and HOMA-IR, 2-hour insulin, $A U C_{\text {INS }}$ and $\Delta I_{30} / \Delta G_{30}$ decreased. Endotheli-
\end{abstract}

\section{KARGER}

E-Mail karger@karger.ch www.karger.com/mpp

\section{(C) 2012 S. Karger AG, Basel} 1011-7571/13/0222-0156\$38.00/0

Karger

0 pen access

This is an Open Access article licensed under the terms of the Creative Commons Attribution- NonCommercial-NoDerivs 3.0 License (www.karger.com/OA-license), applicable to the online version of the article only. Distribution for non-commercial purposes only. um-dependent vasodilation and NO were significantly improved in the treatment group. Furthermore, the changes of endothelium-dependent vasodilation were negatively correlated with changes in $A \cup C_{\text {INS }}$ but positively with $N O$ and HOMA- $\beta$. Stepwise multivariate regression analysis showed that changes in NO and HOMA- $\beta$ were both independent parameters for improvement of endothelial dysfunction. Conclusion: Pioglitazone decreased blood glucose and TG, increased insulin sensitivity, and ameliorated endothelial dysfunction of IGR subjects among the first-degree relatives of T2DM patients. Increased NO production may be associated with the improvement of endothelial dysfunction.

Copyright $\odot 2012$ S. Karger AG, Basel

\section{Introduction}

Diabetic macroangiopathy such as coronary heart disease, stroke and peripheral vascular disease is the leading cause of mortality and disability due to type 2 diabetes mellitus (T2DM). Atherosclerosis is the underlying pathogenesis of macroangiopathy. In the early stage of atherosclerosis, vascular endothelial dysfunction and nitric oxide $(\mathrm{NO})$ synthesis play an important role in the formation and development of atherosclerosis. Studies have shown 
that endothelial dysfunction is detectable in the euglycemic population at high risk for T2DM or subjects with impaired glucose regulation (IGR), including impaired glucose tolerance (IGT) or impaired fasting glucose [1]. Insulin resistance is the common feature of these subjects. Researches indicate that insulin resistance is associated with endothelial dysfunction, independent of age, sex, smoking, body mass index (BMI), body fat and serum lipids [2]. Thiazolidinediones can prevent the development of T2DM by improving insulin resistance, which has been confirmed in several studies [3,4]. It has been reported that rosiglitazone improves the endothelial function in T2DM patients independent of glucose level [5]. Therefore, the aim of this study was to explore whether or not thiazolidinediones can improve the endothelial dysfunction in IGR subjects of the first-degree relatives of T2DM patients and also whether NO plays a role in it.

\section{Subjects and Methods}

\section{Subjects}

The first-degree relatives $(n=107)$ of T2DM patients were selected from the outpatients at the Department of Endocrinology in our hospital from January 2009 to June 2011. The enrolled subjects were 30-59 years of age. They were diagnosed with IGT or impaired fasting glucose for the first time or before but without treatment or already off-treatment for more than 3 months. After 1-month exercise, the subjects were included in the study if they satisfied the following criteria and agreed to change their lifestyle during this study: (a) $5.6 \mathrm{mmol} / \mathrm{l} \leq$ fasting plasma glucose (FPG) $<7.0$ and/or $7.8 \mathrm{mmol} / 1 \leq 2$-hour plasma glucose $(2 \mathrm{hPG})<11.1$ $\mathrm{mmol} / \mathrm{l}$; (b) blood pressure $\leq 140 / 90 \mathrm{~mm} \mathrm{Hg}$; (c) triglyceride (TG) $\leq 4.5 \mathrm{mmol} / \mathrm{l}$, total cholesterol $\leq 7.0 \mathrm{mmol} / \mathrm{l}$; and (d) $\mathrm{BMI}<28$. The selected subjects received $75 \mathrm{~g}$ oral glucose tolerance test and insulin release test. Clinical and laboratory data were collected, including height, weight, blood pressure, brachial artery diameter changes, vascular reactivity, blood NO and other biochemical tests such as serum lipids, liver and renal function, urinalysis and hematology tests. BMI was also calculated. The patients were excluded if they had diabetes mellitus, coronary heart disease, stroke, primary hypertension, chronic obstructive pulmonary disease, hepatic or renal dysfunction or received pharmacological therapy. This clinical study was approved by the Ethics Committee of our hospital. All patients signed the informed consent form.

\section{Methods}

This study was a randomized, double-blind, prospective clinical study. The 107 eligible subjects were randomized to receive pioglitazone $15 \mathrm{mg} /$ day as treatment group $(\mathrm{n}=53$; males: 26 , females: 27$)$ or vehicle as control group ( $n=54$; males: 26 , females: 27). All subjects were followed once a month to evaluate treatment and adverse effects and took part in health education. Telephone follow-up was conducted every half a month to ensure that the subjects adhere to the prescribed amount of exercise. Any missed dose was recorded and added the other day. The study lasted 12 weeks. At the end of the study, the clinical and laboratory tests were repeated.

Blood NO level was determined by nitrate reductase assay (kit provided by Nanjing Juli Biomedical Engineering Institute). Insulin assay was carried out with the Automatic Chemiluminescence System (ACS180 SE) and its kit. Biochemical tests were performed with Roche Modular Biochemical Analyzer and their kits. Coulter STKS hematology analyzer was used to conduct hematological tests.

\section{Measurement of Endothelium-Dependent (EDV) and}

-Independent Vasodilation (EIV)

Color Doppler ultrasonography (ACUSON Sequoia 512, Siemens) was used to measure the inner diameter of the brachial artery at rest, before and after reactive hyperemia, or sublingual nitroglycerin. The subjects stopped smoking for 1 week before this measurement. Thirty-seven females were examined within 1-7 days of menstrual cycle. At the day of examination, the subjects fasted for 8-12 h and the brachial artery segment $2-15 \mathrm{~cm}$ above the elbow was selected for measurement. The longitudinal axis of the brachial artery was scanned. When the arterial intimae of the anterior and posterior walls were clearly seen, the gain was adjusted so that the interface of the lumen could be clearly identified. The ultrasound image was frozen so that the distance between the intimae of the anterior and posterior wall of the brachial artery could be measured when synchronous ECG recording showed the $\mathrm{R}$ wave (end of ventricular systole). The measurements were done at baseline, before and after reactive hyperemia, or sublingual nitroglycerin. The result was calculated as the mean of the three values from three respective cardiac cycles. Patients rested for $10 \mathrm{~min}$ before the test. The baseline brachial artery diameter (D0) was measured before the reactive hyperemia was performed. A blood pressure cuff placed around the arm was inflated to $280 \mathrm{~mm} \mathrm{Hg}$, maintained for $4 \mathrm{~min}$, and then deflated quickly to induce reactive hyperemia. The brachial artery diameter (D1) was measured within 60-90 s after the cuff was released. After 10-15 min, patients were given sublingual nitroglycerin $(0.5 \mathrm{mg})$ and the brachial artery diameter (D2) was measured 5 min later. The ratio of vessel diameter change (\%) was calculated: (D1 or D2 - D0)/D0 $\times 100 \%$.

The maximal percent change of the inner diameter of the brachial artery was calculated as EDV and EIV using the method of Celermajer et al. [6].

\section{Evaluation of $\beta$-Cell Function and Insulin Resistance}

Area under the glucose curve $\left(\mathrm{AUC}_{\mathrm{glu}}=\mathrm{FPG} / 4+\mathrm{PG}_{30} / 2+\right.$ $\mathrm{PG}_{60} / 4+\mathrm{PG}_{120} / 2$, where $\mathrm{PG}$ is plasma glucose, subscript number indicates time) and area under the insulin curve $\left(\mathrm{AUC}_{\mathrm{INS}}\right)$ was calculated in a similar way. Insulin resistance and $\beta$-cell function were evaluated by homeostatic model assessment (HOMA-IR = $\mathrm{FPG} \times \mathrm{FINS}) / 22.5 ; \mathrm{HOMA}-\beta=20 \times \mathrm{FINS} / \mathrm{FPG}-3.5)$ and early insulin secretion index $\left[\Delta \mathrm{I}_{30} / \Delta \mathrm{G}_{30}=\left(\mathrm{INS}_{30}-\mathrm{FINS}\right) /\left(\mathrm{PG}_{30}-\right.\right.$ FPG)] was calculated, where FINS is fasting insulin.

\section{Statistical Analysis}

All data are expressed as mean \pm SEM. Changes in various parameters after treatment with pioglitazone were analyzed using paired test and changes between groups using group $t$ test. Spearman correlation coefficients ( $r$ ) were used to describe the association between variables and a forward stepwise multivariate logistic regression analysis was performed to identify factors that were in- 
Table 1. Comparison of parameters before and after treatment and between the pioglitazone treatment group and the control group

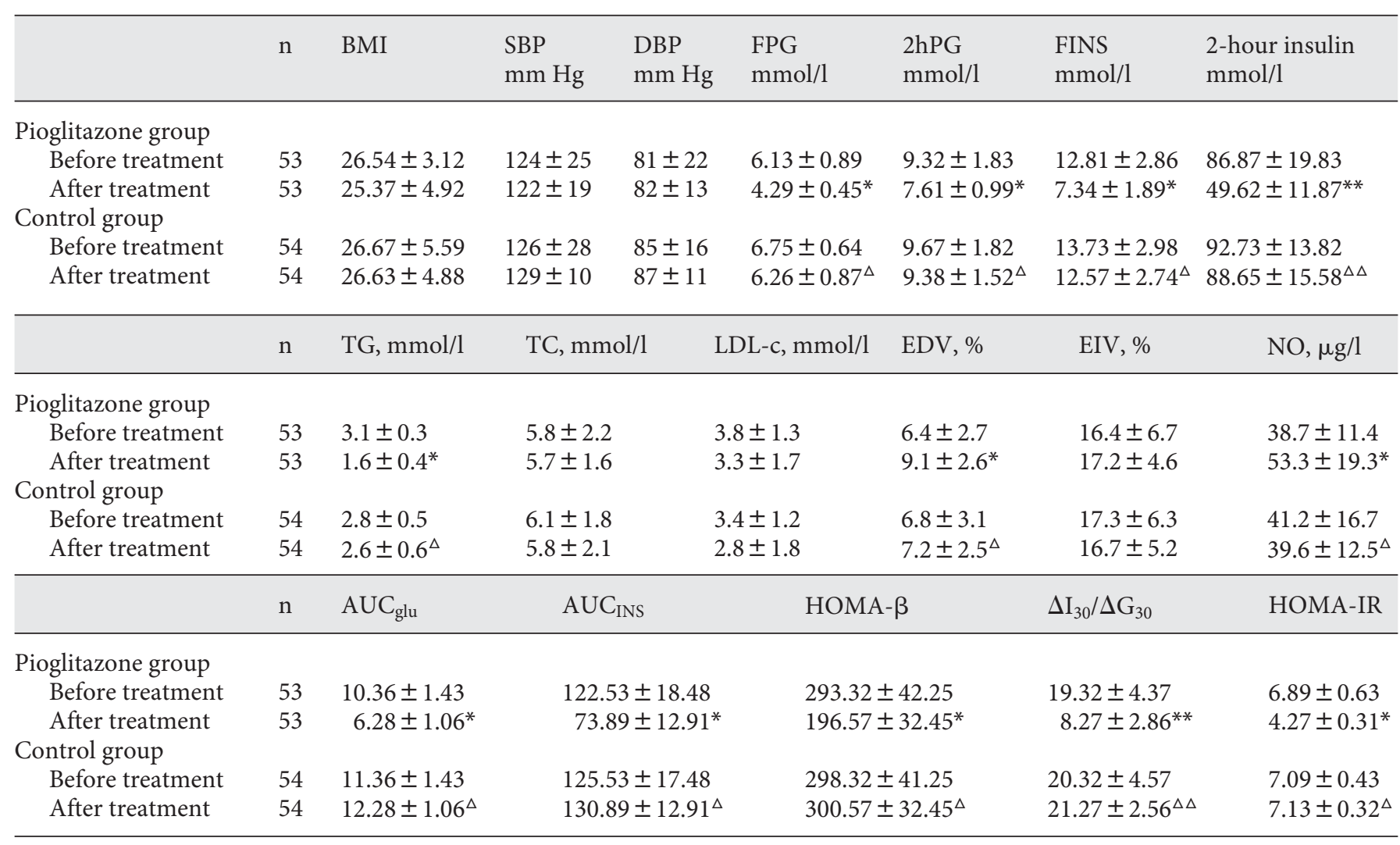

${ }^{*} \mathrm{p}<0.05,{ }^{* *} \mathrm{p}<0.01$ for comparison before and after pioglitazone treatment; ${ }^{\Delta} \mathrm{p}<0.05,{ }^{\Delta \Delta} \mathrm{p}<0.01$ for comparison between pioglitazone and control groups after treatment.

$\mathrm{SBP}=$ Systolic blood pressure; $\mathrm{DBP}=$ diastolic blood pressure; $\mathrm{TC}=$ total cholesterol; LDL-c = low-density lipoprotein cholesterol; $\Delta \mathrm{I}_{30} / \Delta \mathrm{G}_{30}=\left(\mathrm{INS}_{30}-\mathrm{FINS}\right) /\left(\mathrm{PG}_{30}-\mathrm{FPG}\right)$.

dependently associated with a dependent variable. Independent variables included in this model were those that reached statistical significance in Spearman correlation analysis. Statistical significance was defined as $\mathrm{p}<0.05$ (SPSS 10.0 software).

\section{Results}

At baseline, mean age, BMI, systolic and diastolic blood pressure of the study group was $47.3 \pm 7.2$ years, $26.54 \pm 3.12 \mathrm{~kg} / \mathrm{m}^{2}, 124 \pm 25$ and $81 \pm 22 \mathrm{~mm} \mathrm{Hg}$ while that of the control group was $49.5 \pm 9.8$ years, $26.67 \pm$ $5.59 \mathrm{~kg} / \mathrm{m}^{2}, 126 \pm 28$ and $85 \pm 16 \mathrm{~mm} \mathrm{Hg}$. No difference was seen between the two groups in age, BMI, blood pressure and other laboratory characteristics (table 1).

After pioglitazone treatment, FPG, 2hPG, total choles-

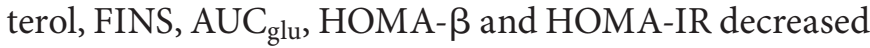
significantly $(\mathrm{p}<0.05)$; 2-hour insulin, $\mathrm{AUC}_{\mathrm{INS}}$ and
$\Delta \mathrm{I}_{30} / \Delta \mathrm{G}_{30}$ also decreased significantly ( $\mathrm{p}<0.01$ ). EDV was improved and blood NO level increased significantly $(p<0.05)$. Compared with the control group, the change of the above parameters was also statistically significant in the treatment group. No significant difference was seen in blood pressure, BMI, EIV, total cholesterol and LDL (table 1).

The simple regression showed that the changes in EDV were negatively correlated with changes in $\mathrm{AUC}_{\mathrm{INS}}$ $(\mathrm{r}=-0.66)$ but positively with $\mathrm{NO}(\mathrm{r}=0.73)$ and HOMA$\beta(r=0.72)$. No significant correlation was present between changes in EDV and TG (table 2). Changes in NO $(\beta=0.62, p=0.022)$ and HOMA- $\beta(\beta=0.59, p=0.036)$ were independent parameters of endothelial dysfunction improvement.

No significant adverse reaction was observed. Liver and renal function tests, urinalysis and hematological tests did not show a significant change after treatment. 
Table 2. Correlations of the changes $(\Delta)$ of EDV and parameters in the pioglitazone group after treatment

\begin{tabular}{lrrrrrrrrrrrr}
\hline Variables & $\Delta \mathrm{BMI}$ & $\Delta \mathrm{SBP}$ & $\Delta \mathrm{DBP}$ & $\Delta \mathrm{TG}$ & $\Delta \mathrm{TC}$ & $\Delta \mathrm{LDL}-\mathrm{c}$ & $\Delta \mathrm{NO}$ & $\Delta \mathrm{AUC}_{\text {glu }}$ & $\Delta \mathrm{AUC}_{\mathrm{INS}} \Delta \mathrm{HOMA}^{-\beta}$ & $\Delta \mathrm{I}_{30} / \Delta \mathrm{G}_{30} \Delta \mathrm{HOMA}^{-\mathrm{IR}}$ \\
\hline $\mathrm{r}$ & -0.20 & -0.36 & -0.39 & -0.42 & -0.18 & -0.22 & 0.73 & -0.11 & -0.66 & 0.72 & 0.24 \\
$\mathrm{p}$ & 0.49 & 0.32 & 0.26 & 0.12 & 0.78 & 0.67 & 0.032 & 0.93 & 0.049 & 0.025 & 0.37 & 0.63 \\
\hline
\end{tabular}

r represents the coefficient of Pearson's correlation.

$\mathrm{SBP}=$ Systolic blood pressure; $\mathrm{DBP}=$ diastolic blood pressure; $\mathrm{TC}=$ total cholesterol; LDL-c = low-density lipoprotein cholesterol; $\Delta \mathrm{I}_{30} / \Delta \mathrm{G}_{30}=\left(\mathrm{INS}_{30}-\mathrm{FINS}\right) /\left(\mathrm{PG}_{30}-\mathrm{FPG}\right)$.

\section{Discussion}

The results showed that the levels of FPG, 2hPG, $\mathrm{AUC}_{\mathrm{glu}}$ and TG decreased. HOMA-IR, FINS, 2-hour insulin, $A U C_{I N S}$, and HOMA- $\beta$, especially $\Delta I_{30} / \Delta G_{30}$ improved significantly. Furthermore, vascular compliance improved and blood NO level increased. These findings suggest that pioglitazone was not only effective in improving the plasma glucose level, serum lipids, and insulin resistance in subjects with IGR among the first-degree relatives of T2DM patients, but also was helpful in improving insulin secretion, endothelial function and increasing blood $\mathrm{NO}$ level.

The treatment regimens for T2DM are updated and modified continually by diabetes associations and societies [7]. However, little effect was observed in the endincidence of diabetic macroangiopathy. Therefore, it is advisable to prevent the development of diabetes at the stage of IGR, including lifestyle intervention and pharmacological therapy such as thiazolidinediones, metformin and acarbose [8]. However, it is not clear whether these measures can also prevent or delay diabetic macroangiopathy in addition to the effects on diabetes prevention.

Diabetic macroangiopathy is closely related to insulin resistance. Recent studies $[9,10]$ indicate that improvement of insulin resistance is associated with significant improvement of endothelial function. Insulin resistance and endothelial dysfunction are present even in prediabetic subjects. Several long-term clinical trials have shown that both IGT and impaired fasting glucose are significant risk factors of death from cardiovascular diseases $[11,12]$. For this reason, it is very interesting to explore if the antidiabetic intervention can prevent the development of both atherosclerosis and T2DM in IGR patients. As seen in this study, pioglitazone treatment decreased blood glucose and improved EDV in IGR. Therefore, pioglitazone may play a role in the prevention of atherosclerosis and T2DM in IGR patients. At present, endothelial dysfunction is the earliest indication of atherosclerosis detectable in a clinical setting [13]. The NO synthesized and released by endothelial cells is crucial in maintaining vascular health and preventing the development of vascular diseases. In this study, correlation analysis showed that changes in EDV were negatively correlated with changes in $\mathrm{AUC}_{\mathrm{INS}}$, but positively with $\mathrm{NO}$ and HOMA- $\beta$ and that changes in NO and HOMA- $\beta$ were independent parameters in improvement of endothelial dysfunction. It suggests that pioglitazone could improve endothelial function via increasing the production of $\mathrm{NO}$ in endothelial cells.

Despite abundant clinical evidence linking metabolic abnormalities to diabetic vasculopathy, the molecular basis of individual susceptibility to diabetic vascular complications is still largely undetermined. Although hyperglycemia, insulin resistance, hyperinsulinemia and dyslipidemia independently contribute to endothelial dysfunction via various mechanisms, the mutual interactions may synergistically accelerate their adverse effects. Oxidative stress and inflammation are predicted to be among the first alterations which may trigger other downstream mediators in diabetes associated with endothelial dysfunction [14]. In prediabetic patients [15, 16], whether with or without CAD, endothelial function was improved after pioglitazone treatment and high molecular weight adiponectin was increased. Hidaka et al. [17] found that pioglitazone, but not amlodipine, improved endothelial function in hypertensive patients with IGT through an increase in NO bioavailability, partly by decreased oxidative stress. In patients with newly diagnosed T2DM, Li et al. [18] reported that intensive insulin therapy could increase serum adiponectin and NO concentrations and improved endothelial function. For the patients with sulfonylureas, Naka et al. [19] found that pioglitazone significantly decreased FINS, HbAlc and HOMA-IR and improved endothelial function, but met- 
formin induced a significant decrease in $\mathrm{HbAlc}$ and only a little improvement in endothelial function which were not associated with the effects of the two insulin sensitizers on glycemic control or insulin resistance. A similar study by Papathanassiou et al. [20] reported that in patients with T2DM already on metformin, addition of pioglitazone as compared to glimepiride improved endothelial function despite similar glycemic control. The improvement in endothelial function was mainly due to a reduction in insulin resistance as HOMA-IR decreased. However, it is not clear whether diabetes period or its treatment contributes to these inconsistent results. Dyslipidemia and endothelial dysfunction both improved in diabetic obese patients after fenofibrate treatment and the endothelial function improvement was significantly associated with the corresponding decrease in apoB-48 (TG-rich lipoprotein) [21].

\section{Conclusion}

Pioglitazone not only decreased blood glucose and TG and increased insulin sensitivity, but also ameliorated endothelial dysfunction of IGR subjects among the firstdegree relatives of T2DM patients. Changes in NO and HOMA- $\beta$ were independent parameters of endothelial dysfunction improvement.

\section{References}

1 Caballero AC: Endothelial dysfunction in obesity and insulin resistance: a road to diabetes and heart disease. Obes Res 2003;11: 1278-1289.

-2 Balletshofer BM, Rittig K, Enderle MD, Volk A, Maerker E, Jacob S, Matthaei S, Rett K, Häring HU: Endothelial dysfunction is detectable in young normotensive first-degree relatives of subjects with type 2 diabetes in association with insulin resistance. Circulation 2000;101:1780-1784.

3 DREAM Trial Investigators, Dagenais GR, Gerstein HC, Holman R, Budaj A, Escalante A, Hedner T, Keltai M, Lonn E, McFarlane S, McQueen M, et al: Effects of ramipril and rosiglitazone on cardiovascular and renal outcomes in people with impaired glucose tolerance or impaired fasting glucose: results of the Diabetes REduction Assessment with ramipril and rosiglitazone Medication (DREAM) trial. Diabetes Care 2008;31: 1007-1014.

4 DeFronzo RA, Tripathy D, Schwenke DC, Banerji M, Bray GA, Buchanan TA, Clement SC, Henry RR, Hodis HN, Kitabchi AE, et al: ACT NOW Study. Pioglitazone for diabetes prevention in impaired glucose tolerance. $\mathrm{N}$ Engl J Med 2011;364:1104-1115.

5 Pistrosch F, Passauer J, Fischer S, Fuecker K, Hanefeld M, Gross P: In type 2 diabetes, rosiglitazone therapy for insulin resistance ameliorates endothelial dysfunction independent of glucose control. Diabetes Care 2004; 27:484-490.

6 Celermajer DS, Sorensen KE, Gooch VW, Sullivan ID, Lloyd JK, Deanfield JE, Spiegelhalter DJ: Non-invasive detection of endothelial dysfunction in children and adults at risk of atherosclerosis. Lancet 1992;340: 1111-1115.

7 Inzucchi SE, Bergenstal RM, Buse JB, Diamant M, Ferrannini E, Nauck M, Peters AL, Tsapas A, Wender R, Matthews DR: Manage- ment of hyperglycaemia in type 2 diabetes: a patient-centered approach. Position statement of the American Diabetes Association (ADA) and the European Association for the Study of Diabetes (EASD). Diabetologia 2012;55:1577-1596.

-8 American Diabetes Association, National Institute of Diabetes and Digestive and Kidney Diseases: Prevention or delay of type 2 diabetes. Diabetes Care 2004;27:s47-s54.

-9 Brame L, Verma S, Anderson T: Insulin resistance as a target for improved endothelial function: metformin. Curr Drug Targets Cardiovasc Haematol 2004;4:53-63.

10 Kim JA, Montagnani M, Koh KK, Quon MJ: Reciprocal relationships between insulin resistance and endothelial dysfunction: molecular and pathophysiological mechanisms. Circulation 2006;113:1888-1904.

11 Saydah SH, Loria CM, Eberhardt MS, Frederick L: Subclinical states of glucose intolerance and risk of death in the U. S. Diabetes Care 2001;24:447-453.

12 Bjornholt JV, Erikssen G, Aaser E, Sandvik L, Nitter-Hauge S, Jervell J, Erikssen J, Thaulow E: Fasting blood glucose: an underestimated risk factor for cardiovascular death: results from a 22-year follow-up of healthy nondiabetic men. Diabetes Care 1999;22:45-49.

13 Baykan M, Erem C, Gedikli O, Hacihasanoglu A, Erdogan T, Kocak M, Kaplan S, Korkmaz L, Celik S: Impairment in flow-mediated vasodilatation of the brachial artery in acromegaly. Med Princ Pract 2009;18:228232.

14 Zhang H, Dellsperger KC, Zhang C: The link between metabolic abnormalities and endothelial dysfunction in type 2 diabetes: an update. Basic Res Cardiol 2012;107:237.

15 Rizza S, Cardellini M, Porzio O, Pecchioli C, Savo A, Cardolini I, Senese N, Lauro D, Sbraccia P, Lauro R, Federici M: Pioglitazone improves endothelial and adipose tissue dysfunction in pre-diabetic CAD subjects. Atherosclerosis 2011;215:180-183.

16 Quinn CE, Lockhart CJ, Hamilton PK, Loughrey CM, McVeigh GE: Effect of pioglitazone on endothelial function in impaired glucose tolerance. Diabetes Obes Metab 2010;12:709-715.

17 Hidaka T, Nakagawa K, Goto C, Soga J, Fujii Y, Hata T, Idei N, Fujimura N, Chayama K, Kihara Y, Higashi Y: Pioglitazone improves endothelium-dependent vasodilation in hypertensive patients with impaired glucose tolerance in part through a decrease in oxidative stress. Atherosclerosis 2010;210:521524

18 Li F, Zhao T, Wen X: Changes in serum adiponectin concentrations and endothelial function after intensive insulin treatment in people with newly diagnosed type 2 diabetes: a pilot study. Diabetes Res Clin Pract 2011; 94:186-192.

19 Naka KK, Papathanassiou K, Bechlioulis A, Pappas K, Kazakos N, Kanioglou C, Kostoula A, Vezyraki P, Makriyiannis D, Tsatsoulis A, Michalis LK: Effects of pioglitazone and metformin on vascular endothelial function in patients with type 2 diabetes treated with sulfonylureas. Diab Vasc Dis Res 2012;9:5258.

20 Papathanassiou K, Naka KK, Kazakos N, Kanioglou C, Makriyiannis D, Pappas K, Katsouras CS, Liveris K, Kolettis T, Tsatsoulis A, Michalis LK: Pioglitazone vs glimepiride: differential effects on vascular endothelial function in patients with type 2 diabetes. Atherosclerosis 2009;205:221-226.

21 Chan DC, Wong AT, Yamashita S, Watts GF: Apolipoprotein B-48 as a determinant of endothelial function in obese subjects with type 2 diabetes mellitus: effect of fenofibrate treatment. Atherosclerosis 2012;221:484489 . 